\title{
Synthesis and reduction reaction of novel triazole compounds in the solid- media condition by using microwave method
}

\author{
Musa Özil a,*, Emre Menteşe a and Bahittin Kahveci b \\ a Department of Chemistry, Faculty of Sciences and Arts, Recep Tayyip Erdoğan University, Rize, TR-53100, Turkey \\ b Department of Nutrition and Dietetics, Faculty of Health Sciences, Karadeniz Technical University, Trabzon, TR-61080, Turkey \\ *Corresponding author at: Department of Chemistry, Faculty of Sciences and Arts, Recep Tayyip Erdoğan University, Rize, TR-53100, Turkey. \\ Tel.: +90.464.2236126; Fax: +90.464.2234019. E-mail address: musaozil@hotmail.com (M. Özil).
}

\section{ARTICLE INFORMATION}

Received: 20 October 2012

Accepted: 28 October 2012

Online: 31 December 2012

\section{KEYWORDS}

Triazole

Reduction

Heterocycles

Synthetic methods

Solid state reactions

Microwave chemistry

\section{Introduction}

Triazol compounds have been found to be associated with diverse pharmacological activities such as antibacterial, antifungal, anticancer, and anticonvulsant [1-5]. Thus, the heterocyclic system is an attractive scaffold to be utilized for exploiting chemical diversity. Microwave-assisted solvent-free reactions have received much attention due to their enhanced reaction rates as well as higher yields and purities. These methods are regarded as environmentally benign and easy-to perform, paving the way to the development of "Green Chemistry" protocols [6-8].

The growing importance of triazole compounds has led to the development of new methods for their synthesis including microwave-assisted synthesis. Thus, the development of simple, convenient, safe and efficient methods for the preparation of these molecules still continues to be an interesting and attractive area of research in synthetic chemistry.

In the present work, we studied the triazole formation, taking different parameters of solid supports like (i) montmorillonite KSF; (ii) montmorillonite K10; (iii) alumina; and (iv) silica gel. However, no reaction occurred. Encouraged by recent focus on the green chemical theme of eliminating the use of solvents, we extended our studies to neat reactions; those that could be made successful without any solid supports. It did not lead to any side reactions and no detectable byproduct was observed. Consequently, we extended this condition to the synthesis of compounds $(\mathbf{2 a - j})$.

\section{Experimental}

\subsection{Instrumentation}

Melting points were recorded in glass capillary tubes, using a Buchi melting point apparatus, and are reported uncorrected. FT-IR spectra were recorded in KBr pellets on a Perkin-Elmer 100 FT-IR spectrophotometer.

${ }^{1} \mathrm{H}$ and ${ }^{13} \mathrm{C}$ NMR spectra were measured on a Varian 400 spectrometer using $\mathrm{CDCl}_{3}$ and DMSO- $d_{6}$ as solvent and TMS as internal standard. Chemical shifts are given in parts per million, coupling constants $J$ in Hertz. Elemental analyses were performed on a Carlo Erba $1106 \mathrm{CHN}$ analyzer.

A multimode Arcelik domestic microwave oven induced reactions were carried out in an open borosil glass vessel under atmospheric pressure. A monomode CEM-Discover Microwave was used in the standard configuration as delivered, including proprietary software. All experiments were carried out in microwave process vials $(30 \mathrm{~mL})$ with control of the temperature by infrared detection temperature sensor. The temperature was computer monitored and maintained constant by a discrete modulation of delivered microwave power. After completion of the reaction, the vial was cooled to $60{ }^{\circ} \mathrm{C}$ by air jet cooling.

\subsection{Synthesis}

2.2.1. General procedure for the synthesis of compounds $2 a-j$ under conventional condition

A mixture of the corresponding ( $N^{\prime}$-ethoxycarbonyl)-4alkyl(aryl)hydrazonic acid ethyl ester (1a-j) $(0.01 \mathrm{~mol})$ and $p$ aminoacetophenone $(0.01 \mathrm{~mol})$ was heated in an oil bath for 5 $\mathrm{h}$ (monitored by TLC, ethylacetate:hexane, $3: 1$ ) at $170{ }^{\circ} \mathrm{C}$. The crystals formed on cooling were recrystallized from ethyl acetate-petroleum ether (3:1) to give pure $\mathbf{2 a - j}$ (Scheme 1). 


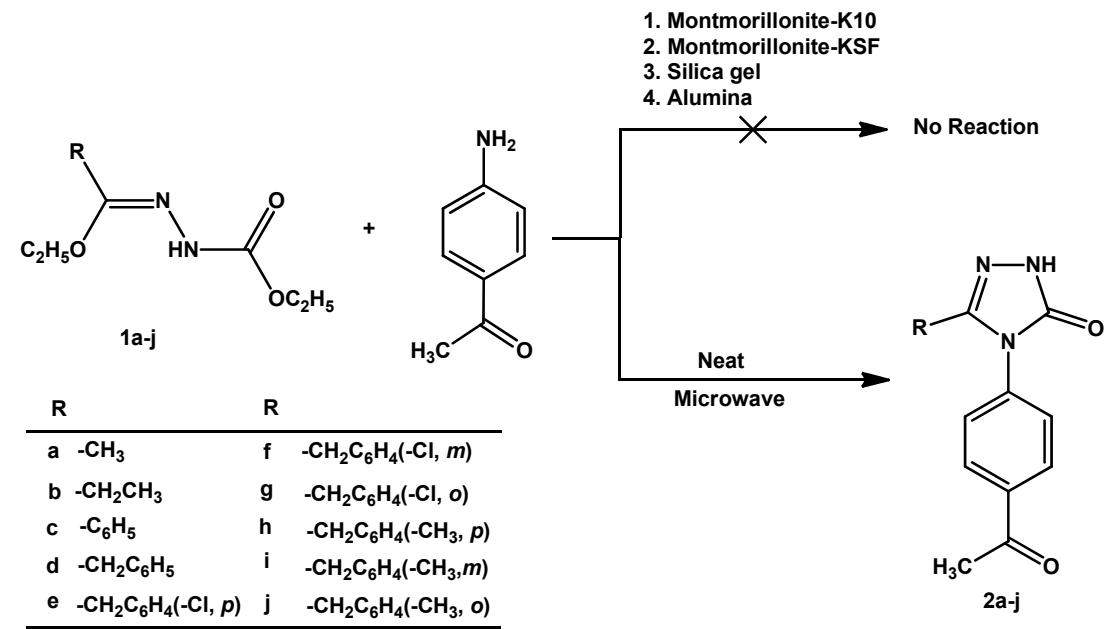

Scheme 1

\subsubsection{General procedure for the synthesis of compounds $2 a-j$ under domestic microwave oven}

A mixture of the corresponding ( $N^{\prime}$-ethoxycarbonyl)-4alkyl(aryl)hydrazonic acid ethyl ester (1a-j) $(0.01 \mathrm{~mol})$ and $p$ aminoacetophenone $(0.01 \mathrm{~mol})$ was added in a flask and the reaction mixture was placed in an alumina bath (neutral alumina) inside a domestic microwave oven and irradiated for $6 \mathrm{~min}$ at $700 \mathrm{~W}$ power. Completion of the reaction was indicated by TLC (ethylacetate:Hexane, 3:1). The crystals formed on cooling were recrystallized from ethyl acetatepetroleum ether (3:1) to give pure $\mathbf{2 a - j}$ (Scheme 1).

\subsubsection{General procedure for the synthesis of compounds $2 a-j$} under monomode Discover (CEM) microwave synthesizer

A mixture of the corresponding ( $N^{\prime}$-ethoxycarbonyl)-4alkyl(aryl)hydrazonic acid ethyl ester (1a-j) $(0.01 \mathrm{~mol})$ and $p$ aminoacetophenone $(0.01 \mathrm{~mol})$ was irradiated in closed vessels with pressure control at $150^{\circ} \mathrm{C}$ for 3 min (hold time) at $300 \mathrm{~W}$ maximum power. After the completion of the reaction (monitored by TLC, AcOEt:hexane, 3:1) the crystals formed on cooling were recrystallized from ethyl acetate-petroleum ether (3:1) to give pure $\mathbf{2 a - j}$ (Scheme 1).

5-Methyl-4-(4-acetylphenyl)-2,4-dihydro-3H-1,2,4-triazol-3one (2a): Yield: $79 \%$. M.p.: $167-168{ }^{\circ} \mathrm{C}$. IR $\left(\mathrm{KBr}, v, \mathrm{~cm}^{-1}\right): 3175$ (NH), 1698 (triazol C=0), 1675 (ket. C=0), $1595(\mathrm{C}=\mathrm{N}) .{ }^{1} \mathrm{H}$ NMR (400 MHz, DMSO- $\left.d_{6}, \delta, \mathrm{ppm}\right): 2.13\left(\mathrm{~s}, 3 \mathrm{H}, \mathrm{CH}_{3}\right), 2.64(\mathrm{~s}, 3 \mathrm{H}$, $\mathrm{CH}_{3}$ ), 7.61 (quasi d, AA' part of AA'XX' system, $J=8.5 \mathrm{~Hz}, 2 \mathrm{H}, \mathrm{Ar}-$ $\mathrm{H}$ ), 8.10 (quasi d, XX' part of AA'XX' system, $J=8.5 \mathrm{~Hz}, 2 \mathrm{H}, \mathrm{Ar}-$ H), 11.77 (s, $1 \mathrm{H}, \mathrm{NH}) .{ }^{13} \mathrm{C}$ NMR (100 MHz, DMSO- $\left.d_{6}, \delta, \mathrm{ppm}\right)$ : 12.43, 26.77, 126.77, 129.14, 136.00, 136.90, 143.38, 153.81, 197.15. Anal. calcd. for $\mathrm{C}_{11} \mathrm{H}_{11} \mathrm{~N}_{3} \mathrm{O}_{2}$ : C, 60.82; $\mathrm{H}, 5.10 ; \mathrm{N}, 19.34$. Found: C: $60.72 ; \mathrm{H}, 5.10 ; \mathrm{N}, 19.11 \%$.

5-Ethyl-4-(4-acetylphenyl)-2,4-dihydro-3H-1,2,4-triazol-3one (2b): Yield: $80 \%$. M.p.: $145-146^{\circ} \mathrm{C}$. IR (KBr, v, $\left.\mathrm{cm}^{-1}\right)$ : 3179 $(\mathrm{NH}), 1710$ (triazol C=0), 1680 (ket. $\mathrm{C}=0), 1574(\mathrm{C}=\mathrm{N}) .{ }^{1} \mathrm{H}$ NMR ( $\left.400 \mathrm{MHz}, \mathrm{DMSO}-d_{6}, \delta, \mathrm{ppm}\right): 1.03\left(\mathrm{t}, J=7.4 \mathrm{~Hz}, 3 \mathrm{H}, \mathrm{CH}_{3}\right), 2.64$ (s, $3 \mathrm{H}, \mathrm{CH}_{3}$ ), $2.48\left(\mathrm{q}, J=7.4 \mathrm{~Hz}, 2 \mathrm{H}, \mathrm{CH}_{2}\right.$ ), 7.60 (quasi d, AA' part of AA'XX' system , $J=8.6 \mathrm{~Hz}, 2 \mathrm{H}, \mathrm{Ar}-\mathrm{H}$ ), 8.10 (quasi d, XX' part of AA'XX' system, $J=8.6 \mathrm{~Hz}, 2 \mathrm{H}, \mathrm{Ar}-\mathrm{H}), 11.77(\mathrm{~s}, 1 \mathrm{H}, \mathrm{NH}) .{ }^{13} \mathrm{C}$ NMR (100 MHz, DMSO- $\left.d_{6}, \delta, \mathrm{ppm}\right): 9.52,19.34,26.78,127.07$, $129.21,136.16,136.12,147.38,154.06,197.15$. Anal. calcd. for $\mathrm{C}_{12} \mathrm{H}_{13} \mathrm{~N}_{3} \mathrm{O}_{2}: \mathrm{C}, 62.33 ; \mathrm{H}, 5.67$; N, 18.17. Found: C: 62.20; H, 5.90; $\mathrm{N}, 18.22 \%$.

5-Phenyl-4-(4-acetylphenyl)-2,4-dihydro-3H-1,2,4-triazol-3one (2c): Yield: $81 \%$. M.p.: $251-252^{\circ} \mathrm{C}$. IR $\left(\mathrm{KBr}, v, \mathrm{~cm}^{-1}\right): 3158$
(NH), 1697 (triazol C=0), 1683 (ket. C=0), $1600(\mathrm{C}=\mathrm{N}) .{ }^{1} \mathrm{H}$ NMR (400 MHz, DMSO- $\left.d_{6}, \delta, \mathrm{ppm}\right): 2.57\left(\mathrm{~s}, 3 \mathrm{H}, \mathrm{CH}_{3}\right), 7.27-7.36(\mathrm{~m}$, $5 \mathrm{H}, \mathrm{Ar}-\mathrm{H}$ ), 7.38 (quasi d, AA' part of AA'XX' system , $J=8.6 \mathrm{~Hz}$, $2 \mathrm{H}, \mathrm{Ar}-\mathrm{H}$ ), 7.99 (quasi d, XX' part of AA'XX' system, $J=8.6 \mathrm{~Hz}$, $2 \mathrm{H}, \mathrm{Ar}-\mathrm{H}), 12.44(\mathrm{~s}, 1 \mathrm{H}, \mathrm{NH}) .{ }^{13} \mathrm{C}$ NMR $\left(100 \mathrm{MHz}, \mathrm{DMSO}-d_{6}, \delta\right.$, ppm): 27.48, 127.58, 128.22, 128.44, 129.31, 129.80, 130.64, $136.85,138.28,145.85,154.85,197.86$. Anal. calcd. for $\mathrm{C}_{16} \mathrm{H}_{13} \mathrm{~N}_{3} \mathrm{O}_{2}$ : C, 68.81; H, 4.69; N, 15.04. Found: C: 68.52; H, 4.91; $\mathrm{N}, 14.94 \%$.

5-Benzyl-4-(4-acetylphenyl)-2,4-dihydro-3H-1,2,4-triazol-3one (2d): Yield: $88 \%$. M.p.: $175-177^{\circ} \mathrm{C}$. IR $\left(\mathrm{KBr}, \mathrm{v}, \mathrm{cm}^{-1}\right): 3171$ $(\mathrm{NH}), 1701$ (triazol C=0), 1688 (ket. $\mathrm{C}=0$ ), $1571(\mathrm{C}=\mathrm{N}) .{ }^{1} \mathrm{H}$ NMR $\left(400 \mathrm{MHz}, \mathrm{DMSO}-d_{6}, \delta, \mathrm{ppm}\right): 2.51\left(\mathrm{~s}, 3 \mathrm{H}, \mathrm{CH}_{3}\right), 3.89(\mathrm{~s}, 2 \mathrm{H}$, $\mathrm{CH}_{2}$ ), 6.98-7.20 (m, 5H, Ar-H), 7.45 (quasi d, AA' part of AA'XX' system , $J=8.2 \mathrm{~Hz}, 2 \mathrm{H}, \mathrm{Ar}-\mathrm{H}$ ), 8.01 (quasi d, XX' part of AA'XX system, $J=8.2 \mathrm{~Hz}, 2 \mathrm{H}, \mathrm{Ar}-\mathrm{H}), 11.90(\mathrm{~s}, 1 \mathrm{H}, \mathrm{NH}) .{ }^{13} \mathrm{C}$ NMR $(100$ MHz, DMSO- $\left.d_{6}, \delta, \mathrm{ppm}\right): 26.78,31.88,126.63,127.22,128.23$, $128.46,129.03,134.79,136.12,136.72,145.48,153.98,197.16$. Anal. calcd. for $\mathrm{C}_{17} \mathrm{H}_{15} \mathrm{~N}_{3} \mathrm{O}_{2}$ : C, 69.61; $\mathrm{H}, 5.15 ; \mathrm{N}, 14.33$. Found: C: 69.49; H, 5.16; N, 14.18\%.

5-(4-Chlorobenzyl)-4-(4-acetylphenyl)-2,4-dihydro-3H-1,2,4triazol-3-one (2e): Yield: 78\%. M.p.: $195-196{ }^{\circ} \mathrm{C}$. IR (KBr, $v, \mathrm{~cm}$ 1): $3171(\mathrm{NH}), 1708$ (triazol C=0), 1688 (ket. $\mathrm{C}=0), 1570(\mathrm{C}=\mathrm{N})$ ${ }^{1} \mathrm{H}$ NMR ( $\left.400 \mathrm{MHz}, \mathrm{CDCl}_{3}, \delta, \mathrm{ppm}\right): 2.62\left(\mathrm{~s}, 3 \mathrm{H}, \mathrm{CH}_{3}\right), 3.80(\mathrm{~s}, 2 \mathrm{H}$, $\mathrm{CH}_{2}$ ), 6.91 (quasi d, AA' part of AA'XX' system , $J=8.2 \mathrm{~Hz}, 2 \mathrm{H}$ $\mathrm{Ar}-\mathrm{H}$ ), 7.17 (quasi d, XX' part of AA'XX' system, $J=8.2 \mathrm{~Hz}, 2 \mathrm{H}$, $\mathrm{Ar}-\mathrm{H}$ ), 7.24 (quasi d, AA' part of AA'XX' system , $J=8.3 \mathrm{~Hz}, 2 \mathrm{H}$ $\mathrm{Ar}-\mathrm{H}$ ), 8.00 (quasi d, XX' part of AA'XX' system, $J=8.3 \mathrm{~Hz}, 2 \mathrm{H}$, $\mathrm{Ar}-\mathrm{H}), 10.63(\mathrm{~s}, 1 \mathrm{H}, \mathrm{NH}) .{ }^{13} \mathrm{C}$ NMR $\left(100 \mathrm{MHz}, \mathrm{CDCl}_{3}, \delta, \mathrm{ppm}\right)$ $26.91,32.34,127.61,129.09,129.84,130.08,132.80,133.60$, $136.49,137.60,146.08,155.26,196.99$. Anal. calcd. for $\mathrm{C}_{17} \mathrm{H}_{14} \mathrm{ClN}_{3} \mathrm{O}_{2}$ : C, 62.30; H, 4.31; N, 12.82. Found: C: 62.23 ; H, $4.41 ; \mathrm{N}, 12.43 \%$.

5-(3-Chlorobenzyl)-4-(4-acetylphenyl)-2,4-dihydro-3H-1,2,4triazol-3-one (2f): Yield: $71 \%$. M.p.: $188-189{ }^{\circ} \mathrm{C}$. IR $(\mathrm{KBr}, v, \mathrm{~cm}$ 1): $3172(\mathrm{NH}), 1703$ (triazol C=0), 1687 (ket. $\mathrm{C}=0), 1573(\mathrm{C}=\mathrm{N})$. ${ }^{1} \mathrm{H}$ NMR (400 MHz, DMSO- $\left.d_{6}, \delta, \mathrm{ppm}\right): 2.63\left(\mathrm{~s}, 3 \mathrm{H}, \mathrm{CH}_{3}\right), 3.93$ (s, $2 \mathrm{H}, \mathrm{CH}_{2}$ ), 7.02-7.23 (m, 4H, Ar-H), 7.49 (quasi d, AA' part of AA'XX' system , $J=9.6 \mathrm{~Hz}, 2 \mathrm{H}, \mathrm{Ar}-\mathrm{H}$ ), 8.04 (quasi d, XX' part of AA'XX' system, $J=9.6 \mathrm{~Hz}, 2 \mathrm{H}, \mathrm{Ar}-\mathrm{H}), 11.93(\mathrm{~s}, 1 \mathrm{H}, \mathrm{NH}) .{ }^{13} \mathrm{C}$ NMR (100 MHz, DMSO- $\left.d_{6}, \delta, \mathrm{ppm}\right): 26.76,31.45,126.62,127.28$, 127.40, 128.67, 129.06, 129.99, 132.65, 136.21, 136.59, 137.09, 145.10, 153.91, 197.11. Anal. calcd. for $\mathrm{C}_{17} \mathrm{H}_{14} \mathrm{ClN}_{3} \mathrm{O}_{2}$ : C, 62.30; H, 4.31; N, 12.82. Found: C: 62.33; H, 4.28; N, 12.81\%.

5-(2-Chlorobenzyl)-4-(4-acetylphenyl)-2,4-dihydro-3H-1,2,4triazol-3-one (2g): Yield: 77\%. M.p.: $197-198{ }^{\circ} \mathrm{C}$. IR (KBr, v, cm 1): $3162(\mathrm{NH}), 1706$ (triazol C=0), 1688 (ket. $\mathrm{C}=0), 1583(\mathrm{C}=\mathrm{N})$. 
${ }^{1} \mathrm{H} \mathrm{NMR}\left(400 \mathrm{MHz}, \mathrm{CDCl}_{3}, \delta, \mathrm{ppm}\right): 2.60\left(\mathrm{~s}, 3 \mathrm{H}, \mathrm{CH}_{3}\right), 3.94(\mathrm{~s}, 2 \mathrm{H}$, $\mathrm{CH}_{2}$ ), 7.14-7.28 (m, 4H, Ar-H), 7.31 (quasi d, AA' part of AA'XX' system , $J=8.5 \mathrm{~Hz}, 2 \mathrm{H}, \mathrm{Ar}-\mathrm{H}$ ), 8.00 (quasi d, XX' part of AA'XX' system, $J=8.5 \mathrm{~Hz}, 2 \mathrm{H}, \mathrm{Ar}-\mathrm{H}), 10.82(\mathrm{~s}, 1 \mathrm{H}, \mathrm{NH}) .{ }^{13} \mathrm{C}$ NMR $(100$ $\left.\mathrm{MHz}_{1} \mathrm{CDCl}_{3}, \delta, \mathrm{ppm}\right): 26.92,30.48,127.08,127.27,127.39$, 129.12, 129.86, 130.70, 132.36, 134.12, 136.52, 137.44, 145.55, 155.31, 197.10. Anal. calcd. for $\mathrm{C}_{17} \mathrm{H}_{14} \mathrm{ClN}_{3} \mathrm{O}_{2}$ : C, 62.30; $\mathrm{H}, 4.31$; $\mathrm{N}, 12.82$. Found: C: $62.41, \mathrm{H}, 4.35 ; \mathrm{N}, 12.76 \%$.

5-(4-Methylbenzyl)-4-(4-acetylphenyl)-2,4-dihydro-3H-1,2,4triazol-3-one (2h): Yield: $72 \%$. M.p.: $171-172{ }^{\circ} \mathrm{C}$. IR (KBr, v, cm1): $3169(\mathrm{NH}), 1716$ (triazol $\mathrm{C}=0), 1681$ (ket. $\mathrm{C}=0), 1588(\mathrm{C}=\mathrm{N})$. ${ }^{1} \mathrm{H}$ NMR $\left(400 \mathrm{MHz}, \mathrm{CDCl}_{3}, \delta, \mathrm{ppm}\right): 2.27\left(\mathrm{~s}, 3 \mathrm{H}, \mathrm{CH}_{3}\right), 2.61(\mathrm{~s}, 3 \mathrm{H}$, $\mathrm{CH}_{3}$ ), $3.78\left(\mathrm{~s}, 2 \mathrm{H}, \mathrm{CH}_{2}\right.$ ), 6.85 (quasi d, $\mathrm{AA}^{\prime}$ part of AA'XX' system, $J=7.9 \mathrm{~Hz}, 2 \mathrm{H}, \mathrm{Ar}-\mathrm{H}$ ), 6.99 (quasi d, XX' part of AA'XX' system, $J=$ $7.9 \mathrm{~Hz}, 2 \mathrm{H}, \mathrm{Ar}-\mathrm{H}$ ), 7.24 (quasi d, AA' part of AA'XX' system , $J=$ $8.6 \mathrm{~Hz}, 2 \mathrm{H}, \mathrm{Ar}-\mathrm{H}$ ), 7.98 (quasi d, XX' part of AA'XX' system, $J=$ $8.6 \mathrm{~Hz}, 2 \mathrm{H}, \mathrm{Ar}-\mathrm{H}), 10.96$ (s, 1H, NH). ${ }^{13} \mathrm{C}$ NMR (100 MHz, $\mathrm{CDCl}_{3}$, $\delta, \mathrm{ppm}): 21.25,26.98,32.52,127.70,128.60,129.60,129.74$, $131.33,136.74,137.18,137.34,146.67,155.52,197.26$. Anal. calcd. for $\mathrm{C}_{18} \mathrm{H}_{17} \mathrm{~N}_{3} \mathrm{O}_{2}$ : C, 70.34; $\mathrm{H}, 5.58$; N, 13.67. Found: C: 70.21; H, 5.61; N, 13.49\%.

5-(3-Methylbenzyl)-4-(4-acetylphenyl)-2,4-dihydro-3H-1,2,4triazol-3-one (2i): Yield: 85\%. M.p.: $139-140{ }^{\circ} \mathrm{C}$. IR (KBr, v, cm1): $3171(\mathrm{NH}), 1710$ (triazol $\mathrm{C}=0), 1687$ (ket. $\mathrm{C}=0), 1571(\mathrm{C}=\mathrm{N})$. ${ }^{1} \mathrm{H}_{\mathrm{NMR}}\left(400 \mathrm{MHz}, \mathrm{DMSO}-d_{6}, \delta, \mathrm{ppm}\right): 2.15\left(\mathrm{~s}, 3 \mathrm{H}, \mathrm{CH}_{3}\right), 2.61(\mathrm{~s}$, $\left.3 \mathrm{H}, \mathrm{CH}_{3}\right), 3.84\left(\mathrm{~s}, 2 \mathrm{H}, \mathrm{CH}_{2}\right), 6.74-7.07(\mathrm{~m}, 4 \mathrm{H}, \mathrm{Ar}-\mathrm{H}), 7.43$ (quasi d, AA' part of AA'XX' system , $J=8.6 \mathrm{~Hz}, 2 \mathrm{H}, \mathrm{Ar}-\mathrm{H}$ ), 8.01 (quasi d, $\mathrm{XX}^{\prime}$ part of AA'XX' system, $\left.J=8.6 \mathrm{~Hz}, 2 \mathrm{H}, \mathrm{Ar}-\mathrm{H}\right), 11.88(\mathrm{~s}, 1 \mathrm{H}$, $\mathrm{NH}) .{ }^{13} \mathrm{C}$ NMR $\left(100 \mathrm{MHz}\right.$, DMSO- $\left.d_{6}, \delta, \mathrm{ppm}\right): 20.71,26.77,31.83$, $125.50,127.23,127.33,128.12,129.00,129.15,134.61,136.18$, $136.75,137.28,145.55,153.98,197.16$. Anal. calcd. for $\mathrm{C}_{18} \mathrm{H}_{17} \mathrm{~N}_{3} \mathrm{O}_{2}: \mathrm{C}, 70.34 ; \mathrm{H}, 5.58 ; \mathrm{N}, 13.67$. Found: $\mathrm{C}: 70.23 ; \mathrm{H}, 5.66$; $\mathrm{N}, 13.71 \%$.

5-(2-Methylbenzyl)-4-(4-acetylphenyl)-2,4-dihydro-3H-1,2,4triazol-3-one (2j): Yield: $78 \%$. M.p.: $183-184{ }^{\circ} \mathrm{C}$. IR (KBr, $v, \mathrm{~cm}$ 1): $3172(\mathrm{NH}), 1701$ (triazol $\mathrm{C}=0), 1681$ (ket. $\mathrm{C}=0), 1573(\mathrm{C}=\mathrm{N})$. ${ }_{1} \mathrm{H}$ NMR ( $\left.400 \mathrm{MHz}, \mathrm{CDCl}_{3}, \delta, \mathrm{ppm}\right): 1.56\left(\mathrm{~d}, J=7.1 \mathrm{~Hz} 3 \mathrm{H}, \mathrm{CH}_{3}\right)$, $2.58\left(\mathrm{~s}, 3 \mathrm{H}, \mathrm{CH}_{3}\right), 3.82\left(\mathrm{q}, J=7.1 \mathrm{~Hz} 2 \mathrm{H}, \mathrm{CH}_{2}\right), 6.89-7.16(\mathrm{~m}, 4 \mathrm{H}$, $\mathrm{Ar}-\mathrm{H}$ ), 7.06 (quasi d, AA' part of AA'XX' system , $J=8.4 \mathrm{~Hz}, 2 \mathrm{H}$, Ar-H), 7.90 (quasi d, XX' part of AA'XX' system, $J=8.4 \mathrm{~Hz}, 2 \mathrm{H}$, $\mathrm{Ar}-\mathrm{H}), 10.96(\mathrm{~s}, 1 \mathrm{H}, \mathrm{NH}) .{ }^{13} \mathrm{C}$ NMR $\left(100 \mathrm{MHz}, \mathrm{CDCl}_{3}, \delta, \mathrm{ppm}\right):$ 20.08, 26.97, 38.10, 127.37, 127.45, 128.14, 128.97, 129.55, $136.71,137.34,141.28,149.80,155.80,197.28$. Anal. calcd. for $\mathrm{C}_{18} \mathrm{H}_{17} \mathrm{~N}_{3} \mathrm{O}_{2}: \mathrm{C}, 70.34 ; \mathrm{H}, 5.58 ; \mathrm{N}, 13.67$. Found: $\mathrm{C}: 70.37 ; \mathrm{H}, 5.50$; $\mathrm{N}, 13.61 \%$.

\subsubsection{General procedure for the synthesis of compounds $3 a-j$ under conventional condition}

A mixture of the $\mathbf{2 a - j}(0.01 \mathrm{~mol}), \mathrm{NaBH}_{4}(0.03 \mathrm{~mol})$ and amberlyst-15 $\left(\mathrm{H}^{+}\right)(5 \mathrm{~g})$ was stirred at room temperature for 12 $\mathrm{h}$ in dry tetrahydrofurane $(100 \mathrm{~mL})$ (monitored by TLC, ethylacetate:Hexane, 3:1). The reaction mixture was filtered. The solvent was removed under reduced pressure. The residue was recrystallized from ethyl acetate-petroleum ether (3:1) to give pure 3a-j (Scheme 2).

\subsubsection{General procedure for the synthesis of compounds $3 a-j$} under domestic microwave oven

A mixture of the $\mathbf{2 a - j}(0.01 \mathrm{~mol}), \mathrm{NaBH}_{4}(0.03 \mathrm{~mol})$ and neutral alumina $(9.5 \mathrm{~g})$ was added in a flask and the reaction mixture was placed in an alumina bath (neutral alumina) inside a domestic microwave oven and irradiated for $4 \mathrm{~min}$ at $1000 \mathrm{~W}$ power. Completion of the reaction was indicated by TLC (ethylacetate:Hexane, 3:1). The reaction mass was eluted with ethanol and $25 \mathrm{~mL}$ of water was added and heated for $10 \mathrm{~min}$. The solvent was removed under reduced pressure. The residue was recrystallized from ethyl acetate-petroleum ether (3:1) to give pure 3a-j (Scheme 2).<smiles>[R]c1n[nH]c(=O)n1-c1ccc(C(C)=O)cc1</smiles>

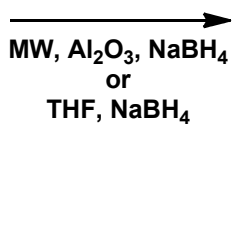<smiles>[R]c1n[nH]c(=O)n1-c1ccc(C(C)O)cc1</smiles>

Scheme 2

2.2.6. General procedure for the synthesis of compounds $3 a-j$ under monomode Discover (CEM) microwave synthesizer

A mixture of the $2 \mathbf{2 a}-\mathbf{j}(0.01 \mathrm{~mol}), \mathrm{NaBH}_{4}(0.03 \mathrm{~mol})$ and neutral alumina $(9.5 \mathrm{~g})$ was added in a pyrex open vessel (30 $\mathrm{mL}$ ) and microwave irradiated at $150{ }^{\circ} \mathrm{C}$ for 2 min (hold time) at $300 \mathrm{~W}$ maximum power. Completion of the reaction was indicated by TLC (ethylacetate:Hexane, 3:1). The reaction mass was eluted with ethanol and $25 \mathrm{~mL}$ of water was added and heated for $10 \mathrm{~min}$. The solvent was removed under reduced pressure. The residue was recrystallized from ethyl acetatepetroleum ether (3:1) to give pure $\mathbf{3 a - j}$ (Scheme 2).

5-Methyl-4-(4-[1-hydroxyetyl]phenyl)-2,4-dihydro-3H-1,2,4triazol-3-one (3a): Yield=67\%. M.p.: $148-149^{\circ} \mathrm{C}$. IR $(\mathrm{KBr}, v, \mathrm{~cm}$ 1): $3303(\mathrm{OH}), 3192(\mathrm{NH}), 1692(\mathrm{C}=0), 1598(\mathrm{C}=\mathrm{N}), 1088(\mathrm{C}-0)$. ${ }^{1} \mathrm{H}$ NMR $\left(400 \mathrm{MHz}\right.$, DMSO- $\left.d_{6}, \delta, \mathrm{ppm}\right): 1.34(\mathrm{~d}, J=6.4 \mathrm{~Hz}, 3 \mathrm{H}$, $\left.\mathrm{CH}_{3}\right), 2.02\left(\mathrm{~s}, 3 \mathrm{H}, \mathrm{CH}_{3}\right), 4.76(\mathrm{dq}, J=6.4 \mathrm{~Hz}, 1 \mathrm{H}, \mathrm{CH}), 5.23(\mathrm{~d}, J=$ $3.8 \mathrm{~Hz}, 1 \mathrm{H}, \mathrm{OH}$ ), 7.31 (quasi d, AA' part of AA'XX' system , $J=8.4$ $\mathrm{Hz}, 2 \mathrm{H}, \mathrm{Ar}-\mathrm{H}$ ), 7.46 (quasi d, XX' part of AA'XX' system, $J=8.4$ $\mathrm{Hz}, 2 \mathrm{H}, \mathrm{Ar}-\mathrm{H}), 11.53$ (s, 1H, NH). ${ }^{13} \mathrm{C}$ NMR $\left(100 \mathrm{MHz}, \mathrm{DMSO}-d_{6}\right.$, $\delta, \mathrm{ppm}): 13.00,26.52,68.33,126.85,127.40,132.02,144.52$, 148.34, 155.04. Anal. calcd. for $\mathrm{C}_{11} \mathrm{H}_{13} \mathrm{~N}_{3} \mathrm{O}_{2}$ : C, 60.26; H, 5.98; N, 19.17. Found: C: $60.13 ; \mathrm{H}, 5.90 ; \mathrm{N}, 19.18 \%$.

5-Ethyl-4-(4-[1-hydroxyetyl]phenyl)-2,4-dihydro-3H-1,2,4triazol-3-one (3b): Yield $=61 \%$. M.p.: $115-116^{\circ} \mathrm{C}$. IR $(\mathrm{KBr}, v, \mathrm{~cm}$ 1): $3294(\mathrm{OH}), 3183(\mathrm{NH}), 1697$ (C=0), 1592 (C=N), 1090 (C-O). ${ }_{1}^{1} \mathrm{H}$ NMR $\left(400 \mathrm{MHz}\right.$, DMSO- $\left.d_{6}, \delta, \mathrm{ppm}\right): 1.00(\mathrm{t}, J=7.4 \mathrm{~Hz}, 3 \mathrm{H}$, $\mathrm{CH}_{3}$ ), 1.33 (d, $\left.J=6.6 \mathrm{~Hz}, 3 \mathrm{H}, \mathrm{CH}_{3}\right), 2.36\left(\mathrm{q}, J=7.4 \mathrm{~Hz}, 2 \mathrm{H}, \mathrm{CH}_{2}\right.$ ), $4.77(\mathrm{dq}, J=6.6 \mathrm{~Hz}, 1 \mathrm{H}, \mathrm{CH}), 5.28(\mathrm{~d}, J=4.3 \mathrm{~Hz}, 1 \mathrm{H}, \mathrm{OH}), 7.30$ (quasi d, AA' part of AA'XX' system , $J=8.4 \mathrm{~Hz}, 2 \mathrm{H}, \mathrm{Ar}-\mathrm{H}$ ), 7.45 (quasi d, XX' part of AA'XX' system, $J=8.4 \mathrm{~Hz}, 2 \mathrm{H}, \mathrm{Ar}-\mathrm{H}$ ), 11.58 $(\mathrm{s}, 1 \mathrm{H}, \mathrm{NH}) .{ }^{13} \mathrm{C}$ NMR $\left(100 \mathrm{MHz}, \mathrm{DMSO}-d_{6}, \delta, \mathrm{ppm}\right): 10.42,19.98$, $26.49,68.33,126.91,127.64,131.99,148.47,148.59,155.30$. Anal. calcd. for $\mathrm{C}_{12} \mathrm{H}_{15} \mathrm{~N}_{3} \mathrm{O}_{2}$ : C, 61.79; H, 6.48; N, 18.01. Found: C: $61.75 ; \mathrm{H}, 6.40 ; \mathrm{N}, 18.05 \%$.

5-Phenyl-4-(4-[1-hydroxyetyl]phenyl)-2,4-dihydro-3H-1,2,4triazol-3-one (3c): Yield= 73\%. M.p.: $192-193^{\circ} \mathrm{C}$. IR $(\mathrm{KBr}, v, \mathrm{~cm}$ 1): $3414(\mathrm{OH}), 3197(\mathrm{NH}), 1701(\mathrm{C}=0), 1515(\mathrm{C}=\mathrm{N}), 1089(\mathrm{C}-\mathrm{O})$. ${ }^{1} \mathrm{H}$ NMR (400 MHz, DMSO- $\left.d_{6}, \delta, \mathrm{ppm}\right): 1.33(\mathrm{~d}, J=6.6 \mathrm{~Hz}, 3 \mathrm{H}$ $\left.\mathrm{CH}_{3}\right), 4.76(\mathrm{dq}, J=6.6 \mathrm{~Hz}, 1 \mathrm{H}, \mathrm{CH}), 5.30(\mathrm{~d}, J=4.4 \mathrm{~Hz}, 1 \mathrm{H}, \mathrm{OH})$, 7.27-7.38 (m, 5H, Ar-H), 7.20 (quasi d, AA' part of AA'XX' system , $J=8.6 \mathrm{~Hz}, 2 \mathrm{H}, \mathrm{Ar}-\mathrm{H}$ ), 7.41 (quasi d, XX' part of AA'XX system, $J=8.6 \mathrm{~Hz}, 2 \mathrm{H}, \mathrm{Ar}-\mathrm{H}), 12.17(\mathrm{~s}, 1 \mathrm{H}, \mathrm{NH}) .{ }^{13} \mathrm{C}$ NMR $(100$ MHz, DMSO- $\left.d_{6}, \delta, \mathrm{ppm}\right): 26.51,68.24,126.79,127.83,127.99$, $128.29,129.19,130.46,132.65,146.12,148.40,155.35$. Anal. calcd. for $\mathrm{C}_{16} \mathrm{H}_{15} \mathrm{~N}_{3} \mathrm{O}_{2}$ : C, 68.31; $\mathrm{H}, 5.37 ; \mathrm{N}, 14.94$. Foun: C 68.40; $\mathrm{H}, 5.33$; N, $14.67 \%$.

5-Benzyl-4-(4-[1-hydroxyetyl]phenyl)-2,4-dihydro-3H-1,2,4triazol-3-one (3d): Yield: $69 \%$. M.p.: $156-157^{\circ} \mathrm{C}$. IR (KBr, $v, \mathrm{~cm}$ 1): $3359(\mathrm{OH}), 3183(\mathrm{NH}), 1701(\mathrm{C}=0), 1591(\mathrm{C}=\mathrm{N}), 1088(\mathrm{C}-\mathrm{O})$. ${ }^{1} \mathrm{H} \mathrm{NMR}\left(400 \mathrm{MHz}, \mathrm{CDCl}_{3}, \delta, \mathrm{ppm}\right): 1.47\left(\mathrm{~d}, J=6.5 \mathrm{~Hz}, 3 \mathrm{H}, \mathrm{CH}_{3}\right)$, 2.61 (bs, 1H, OH), 3.77 (s, 2H, CH $), 4.90$ (q, J = $6.5 \mathrm{~Hz}, 1 \mathrm{H}, \mathrm{CH}$ ), 6.94-7.26 (m, 5H, Ar-H), 7.03 (quasi d, AA' part of $\mathrm{AA}^{\prime} \mathrm{XX}^{\prime}$ system , $J=8.4 \mathrm{~Hz}, 2 \mathrm{H}, \mathrm{Ar}-\mathrm{H}$ ), 7.38 (quasi d, XX' part of AA'XX' system, $J=8.4 \mathrm{~Hz}, 2 \mathrm{H}, \mathrm{Ar}-\mathrm{H}), 10.58(\mathrm{~s}, 1 \mathrm{H}, \mathrm{NH}) .{ }^{13} \mathrm{C}$ NMR $(100$ $\left.\mathrm{MHz}, \mathrm{CDCl}_{3}, \delta, \mathrm{ppm}\right): 25.62,32.82,69.88,126.74,127.35$, 
$127.76,128.76,128.83,131.42,134.70,147.19,147.42,155.78$. Anal. calcd. for $\mathrm{C}_{17} \mathrm{H}_{17} \mathrm{~N}_{3} \mathrm{O}_{2}$ : C, 69.14; $\mathrm{H}, 5.80 ; \mathrm{N}, 14.23$. Found: C: 69.22; H, 5.81; N, 14.29\%.

5-(4-Chlorobenzyl)-4-(4-[1-hydroxyetyl]phenyl)-2,4-dihydro3H-1,2,4-triazol-3-one (3e): Yield: $75 \%$. M.p.: $160-161{ }^{\circ} \mathrm{C}$. IR $\left(\mathrm{KBr}, v, \mathrm{~cm}^{-1}\right): 3306(\mathrm{OH}), 3182(\mathrm{NH}), 1700(\mathrm{C}=0), 1591(\mathrm{C}=\mathrm{N})$, 1087 (C-0). ${ }^{1} \mathrm{H}$ NMR (400 MHz, $\left.\mathrm{CDCl}_{3}, \delta, \mathrm{ppm}\right): 1.48(\mathrm{~d}, J=6.5$ $\left.\mathrm{Hz}, 3 \mathrm{H}, \mathrm{CH}_{3}\right), 2.56(\mathrm{bs}, 1 \mathrm{H}, \mathrm{OH}), 3.73\left(\mathrm{~s}, 2 \mathrm{H}, \mathrm{CH}_{2}\right), 4.91(\mathrm{q}, J=6.5$ $\mathrm{Hz}, 1 \mathrm{H}, \mathrm{CH}$ ), 6.89 (quasi d, AA' part of AA'XX' system , $J=8.4 \mathrm{~Hz}$, $2 \mathrm{H}, \mathrm{Ar}-\mathrm{H}$ ), 7.15 (quasi d, XX' part of AA'XX' system, $J=8.4 \mathrm{~Hz}$, $2 \mathrm{H}, \mathrm{Ar}-\mathrm{H}$ ), 7.05 (quasi d, AA' part of AA'XX' system , $J=8.4 \mathrm{~Hz}$, $2 \mathrm{H}, \mathrm{Ar}-\mathrm{H}$ ), 7.40 (quasi d, XX' part of AA'XX' system, $J=8.4 \mathrm{~Hz}$, $2 \mathrm{H}, \mathrm{Ar}-\mathrm{H}), 10.59$ (s, $1 \mathrm{H}, \mathrm{NH}) .{ }^{13} \mathrm{C}$ NMR (100 MHz, $\left.\mathrm{CDCl}_{3}, \delta, \mathrm{ppm}\right)$ : 25.66, 32.21, 69.85, 126.85, 127.69, 128.92, 130.21, 131.31, $133.11,133.34,146.73,147.58,155.69$. Anal. calcd. for $\mathrm{C}_{17} \mathrm{H}_{16} \mathrm{ClN}_{3} \mathrm{O}_{2}$ : C, 61.91; H, 4.89; N, 12.74. Found: C: 61.80; H, $4.81 ; \mathrm{N}, 12.70 \%$.

5-(3-Chlorobenzyl)-4-(4-[1-hydroxyetyl]phenyl)-2,4-dihydro3H-1,2,4-triazol-3-one (3f): Yield: $66 \%$. M.p.: $152-153{ }^{\circ} \mathrm{C}$. IR $\left(\mathrm{KBr}, \mathrm{v}, \mathrm{cm}^{-1}\right): 3306(\mathrm{OH}), 3183(\mathrm{NH}), 1701(\mathrm{C}=0), 1591(\mathrm{C}=\mathrm{N})$, $1088(\mathrm{C}-0) .{ }^{1} \mathrm{H}$ NMR $\left(400 \mathrm{MHz}, \mathrm{DMSO}-d_{6}, \delta, \mathrm{ppm}\right): 1.34(\mathrm{~d}, J=$ $\left.6.2 \mathrm{~Hz}, 3 \mathrm{H}, \mathrm{CH}_{3}\right), 3.82\left(\mathrm{~s}, 2 \mathrm{H}, \mathrm{CH}_{2}\right), 4.77(\mathrm{~d}, J=6.2 \mathrm{~Hz}, 1 \mathrm{H}, \mathrm{CH})$, 5.30 (bs, $1 \mathrm{H}, \mathrm{OH}$ ), 7.19-7.23 (m, 4H, Ar-H), 7.01 (quasi d, AA' part of AA'XX' system , $J=7.6 \mathrm{~Hz}, 2 \mathrm{H}, \mathrm{Ar}-\mathrm{H}$ ), 7.42 (quasi d, XX' part of AA'XX' system, $J=7.6 \mathrm{~Hz}, 2 \mathrm{H}, \mathrm{Ar}-\mathrm{H}), 11.78(\mathrm{~s}, 1 \mathrm{H}, \mathrm{NH})$. ${ }^{13} \mathrm{C}$ NMR (100 MHz, DMSO- $\left.d_{6}, \delta, \mathrm{ppm}\right): 25.90,31.39,67.52$, $125.96,126.60,127.01,127.41,128.64,129.95,130.83,132.66$, $137.33,145.52,147.88,154.36$. Anal. calcd. for $\mathrm{C}_{17} \mathrm{H}_{16} \mathrm{ClN}_{3} \mathrm{O}_{2}$ : C, 61.91; H, 4.89; N, 12.74. Found: C: 61.90; H, 4.84; N, $12.77 \%$.

5-(2-Chlorobenzyl)-4-(4-[1-hydroxyetyl]phenyl)-2,4-dihydro3H-1,2,4-triazol-3-one (3g): Yield: $78 \%$. M.p.: $219-220{ }^{\circ} \mathrm{C}$. IR $\left(\mathrm{KBr}, v, \mathrm{~cm}^{-1}\right): 3426(\mathrm{OH}), 3186(\mathrm{NH}), 1691(\mathrm{C}=0), 1575(\mathrm{C}=\mathrm{N})$, $1089(\mathrm{C}-0) .{ }^{1} \mathrm{H}$ NMR (400 MHz, DMSO- $\left.d_{6}, \delta, \mathrm{ppm}\right): 1.31(\mathrm{~d}, J=$ $\left.5.6 \mathrm{~Hz}, 3 \mathrm{H}, \mathrm{CH}_{3}\right), 3.84\left(\mathrm{~s}, 2 \mathrm{H}, \mathrm{CH}_{2}\right), 4.75$ (bs, $\left.1 \mathrm{H}, \mathrm{CH}\right), 5.25(\mathrm{~s}, 1 \mathrm{H}$, $\mathrm{OH}), 7.21-7.43(\mathrm{~m}, 8 \mathrm{H}, \mathrm{Ar}-\mathrm{H}), 11.69(\mathrm{~s}, 1 \mathrm{H}, \mathrm{NH}) .{ }^{13} \mathrm{C}$ NMR $(100$ MHz, DMSO- $\left.d_{6}, \delta, \mathrm{ppm}\right): 26.57,30.64,68.30,126.87,127.67$, $127.79,129.45,129.79,131.68,131.95,133.58,133.86,145.59$, 148.68, 155.12. Anal. calcd. for $\mathrm{C}_{17} \mathrm{H}_{16} \mathrm{ClN}_{3} \mathrm{O}_{2}$ : C, 61.91; $\mathrm{H}, 4.89$; N, 12.74. Found: C: $61.90 ; \mathrm{H}, 4.81, \mathrm{~N}, 12.77 \%$.

5-(4-Methylbenzyl)-4-(4-[1-hydroxyetyl]phenyl)-2,4-dihydro3H-1,2,4-triazol-3-one (3h): Yield: $75 \%$. M.p.: $127-128{ }^{\circ} \mathrm{C}$. IR $\left(\mathrm{KBr}, v, \mathrm{~cm}^{-1}\right): 3420(\mathrm{OH}), 3177(\mathrm{NH}), 1702(\mathrm{C}=0), 1577(\mathrm{C}=\mathrm{N})$, 1090 (C-0). ${ }^{1} \mathrm{H}$ NMR (400 MHz, $\left.\mathrm{CDCl}_{3}, \delta, \mathrm{ppm}\right): 1.48(\mathrm{~d}, J=6.5$ $\left.\mathrm{Hz}, 3 \mathrm{H}, \mathrm{CH}_{3}\right), 2.28\left(\mathrm{~s}, 3 \mathrm{H}, \mathrm{CH}_{3}\right), 2.43(\mathrm{bs}, 1 \mathrm{H}, \mathrm{OH}), 3.72(\mathrm{~s}, 2 \mathrm{H}$, $\left.\mathrm{CH}_{2}\right), 4.92(\mathrm{q}, J=6.5 \mathrm{~Hz}, 1 \mathrm{H}, \mathrm{CH}$ ), 6.85 (quasi d, AA' part of AA'XX' system , $J=8.0 \mathrm{~Hz}, 2 \mathrm{H}, \mathrm{Ar}-\mathrm{H}$ ), 7.00 (quasi d, XX' part of AA'XX' system, $J=8.0 \mathrm{~Hz}, 2 \mathrm{H}, \mathrm{Ar}-\mathrm{H}$ ), 7.06 (quasi d, AA' part of AA'XX' system , $J=8.4 \mathrm{~Hz}, 2 \mathrm{H}, \mathrm{Ar}-\mathrm{H}$ ), 7.40 (quasi d, $\mathrm{XX}^{\prime}$ part of $\mathrm{AA}^{\prime} \mathrm{XX}$ ' system, $\left.J=8.4 \mathrm{~Hz}, 2 \mathrm{H}, \mathrm{Ar}-\mathrm{H}\right), 10.34(\mathrm{~s}, 1 \mathrm{H}, \mathrm{NH}) .{ }^{13} \mathrm{C}$ NMR (100 MHz, $\left.\mathrm{CDCl}_{3}, \delta, \mathrm{ppm}\right): 21.22,25.60,32.43,69.94,126.72$, $127.78,128.72,129.46,131.56,136.99,147.32,147.42,155.67$. Anal. calcd. for $\mathrm{C}_{18} \mathrm{H}_{19} \mathrm{~N}_{3} \mathrm{O}_{2}$ : C, 69.88; H, 6.19; N, 13.58. Found: C: 69.85; H, 6.21; N, 13.54\%.

5-(3-Methylbenzyl)-4-(4-[1-hydroxyetyl]phenyl)-2,4-dihydro3H-1,2,4-triazol-3-one (3i): Yield: 69\%. M.p.: $156-158{ }^{\circ} \mathrm{C}$. IR $\left(\mathrm{KBr}, v, \mathrm{~cm}^{-1}\right): 3419(\mathrm{OH}), 3168(\mathrm{NH}), 1705(\mathrm{C}=0), 1578(\mathrm{C}=\mathrm{N})$, 1088 (C-0). ${ }^{1} \mathrm{H}$ NMR ( $\left.400 \mathrm{MHz}, \mathrm{CDCl}_{3}, \delta, \mathrm{ppm}\right): 1.46$ (d, $J=6.4$ $\left.\mathrm{Hz}, 3 \mathrm{H}, \mathrm{CH}_{3}\right), 2.21\left(\mathrm{~s}, 3 \mathrm{H}, \mathrm{CH}_{3}\right), 3.07(\mathrm{~s}, 1 \mathrm{H}, \mathrm{OH}), 3.72(\mathrm{~s}, 2 \mathrm{H}$, $\left.\mathrm{CH}_{2}\right), 4.88(\mathrm{q}, J=6.4 \mathrm{~Hz}, 1 \mathrm{H}, \mathrm{CH}), 6.72-7.07(\mathrm{~m}, 4 \mathrm{H}, \mathrm{Ar}-\mathrm{H}), 7.01$ (quasi d, AA' part of AA'XX' system , $J=8.2 \mathrm{~Hz}, 2 \mathrm{H}, \mathrm{Ar}-\mathrm{H}$ ), 7.37 (quasi d, XX' part of AA'XX' system, $J=8.2 \mathrm{~Hz}, 2 \mathrm{H}, \mathrm{Ar}-\mathrm{H}$ ), 10.90 (s, 1H, NH). ${ }^{13} \mathrm{C}$ NMR $\left(100 \mathrm{MHz}, \mathrm{CDCl}_{3}, \delta, \mathrm{ppm}\right): 21.47,25.59$, $32.73,69.78,125.90,126.68,127.79,128.04,128.62,129.63$, $131.42,134.60,138.37,147.27,147.48,155.89$. Anal. calcd. for $\mathrm{C}_{18} \mathrm{H}_{19} \mathrm{~N}_{3} \mathrm{O}_{2}: \mathrm{C}, 69.88 ; \mathrm{H}, 6.19$; N, 13.58. Found: C: 69.75; H, 6.21; $\mathrm{N}, 13.53 \%$.

5-(2-Methylbenzyl)-4-(4-[1-hydroxyetyl]phenyl)-2,4-dihydro3H-1,2,4-triazol-3-one (3j): Yield: 75\%. M.p.: 143-145 ${ }^{\circ} \mathrm{C}$. IR $\left(\mathrm{KBr}, v, \mathrm{~cm}^{-1}\right): 3474(\mathrm{OH}), 3184(\mathrm{NH}), 1697(\mathrm{C}=0), 1571(\mathrm{C}=\mathrm{N})$, 1089 (C-O). ${ }^{1} \mathrm{H}$ NMR (400 MHz, $\left.\mathrm{CDCl}_{3}, \delta, \mathrm{ppm}\right): 1.46$ (d, $J=6.5$ $\left.\mathrm{Hz}, 3 \mathrm{H}, \mathrm{CH}_{3}\right), 1.55\left(\mathrm{~d}, J=7.2 \mathrm{~Hz}, 3 \mathrm{H}, \mathrm{CH}_{3}\right), 2.40(\mathrm{~s}, 1 \mathrm{H}, \mathrm{OH}), 3.79$ $\left(\mathrm{dq}, J=7.2 \mathrm{~Hz}, 2 \mathrm{H}, \mathrm{CH}_{2}\right), 4.88(\mathrm{q}, J=6.5 \mathrm{~Hz}, 1 \mathrm{H}, \mathrm{CH}), 6.86-7.16$ $(\mathrm{m}, 4 \mathrm{H}, \mathrm{Ar}-\mathrm{H}$ ), 7.16 (quasi d, AA' part of AA'XX' system , $J=8.4$ $\mathrm{Hz}, 2 \mathrm{H}, \mathrm{Ar}-\mathrm{H}$ ), 7.31 (quasi d, XX' part of AA'XX' system, $J=8.4$ $\mathrm{Hz}, 2 \mathrm{H}, \mathrm{Ar}-\mathrm{H}), 10.46$ (s, $1 \mathrm{H}, \mathrm{NH}) .{ }^{13} \mathrm{C}$ NMR $\left(100 \mathrm{MHz}, \mathrm{CDCl}_{3}, \delta\right.$, ppm): 19.99, 25.62, 37.91, 69.91, 126.52, 127.27, 127.44, 128.07, 128.08, 128.81, 131.42, 141.52, 147.28, 150.51, 156.02. Anal. calcd. for $\mathrm{C}_{18} \mathrm{H}_{19} \mathrm{~N}_{3} \mathrm{O}_{2}: \mathrm{C}, 69.88 ; \mathrm{H}, 6.19 ; \mathrm{N}, 13.58$. Found: C: 69.91; H, 6.22; N, 13.44\%.

\section{Results and discussion}

Recently, we reported the synthesis of triazole compounds by both microwave irradiation and conventional heating $[9,10]$ However low reaction yields, selectivity and long reaction times dissuade from the more systematic study of the reaction. Domestic microwave ovens, in particular, have caused the solvent to explode. Therefore, we have planned to focus our study on the synthesis and reduction of triazole compounds via conventional as well as microwave-assisted synthesis under solvent free media. ( $N^{\prime}$-ethoxycarbonyl)-4-alkyl(aryl) hydrazonic acid ethyl ester can be considered as useful intermediate leading to the formation of heterocycles such as 1,2,4-triazole3 -ones. We synthesized some ( $N^{\prime}$-ethoxycarbonyl)-4-alkyl(aryl) hydrazonic acid ethyl ester (1a-j) according to literature [1012].

At first, we had to optimize the reaction conditions for the synthesis of 5-aryl(alkyl)-4-(4-acetylphenyl)-2,4-dihydro-3 $\mathrm{H}$ 1,2,4-triazol-3-one (2a-j) by the reaction of ( $N^{\prime}$-ethoxycarbonyl)-4-alkyl(aryl)hydrazonic acid ethyl ester (1a-j) with $p$-aminoacetophenone. We tried different reaction conditions to carry out this reaction under microwave irradiation in the absence of solvent. For this aim we used solid support, such as montmorillonite KSF, montmorillonite K10, alumina and silica gel, but no reaction occurred. However, reaction occurred when heated neat above their melting points under microwave irradiation (Scheme 1).

This case of reaction is propitious to the observation of specific microwave (MW) effects when considering the mechanism. The polarity of the system is increased during the reaction progress from the neutral ground state (GS) to the dipolar transition state (TS) for cyclization. Consequently, as MW stabilizing effects by dipole-dipole interactions with the electric field are increased with the polarity of reactants. Such an explanation of MW effects is supported by calculations suggesting a relation between MW irradiation and the polarity of the transition state [13]. So we can say in our reactions, specific MW effects occur when the reaction mixture is melting.

Encouraged by these findings, we next started to investigate the optimum reaction condition for the synthesis of $3 H-1,2,4$-triazol-3-ones (2a-j) under conventional conditions and Discover (CEM) monomodal microwave oven and multimode microwave oven. For conventional conditions the reaction occurred in an oil bath for $5 \mathrm{~h}$ at $170{ }^{\circ} \mathrm{C}$. For Discover (CEM) monomodal microwave oven, mixture was irradiated in closed vessels with pressure control at $150{ }^{\circ} \mathrm{C}$ for $3 \mathrm{~min}$ (hold time) at $300 \mathrm{~W}$ maximum power. For the domestic microwave oven, the mixture was irradiated in a flask in an alumina bath for $12 \mathrm{~min}, 9 \mathrm{~min}, 6 \mathrm{~min}$ and $4 \mathrm{~min}$ at $350 \mathrm{~W}, 500 \mathrm{~W}, 700 \mathrm{~W}$ and $1000 \mathrm{~W}$ power, respectively. As alumina is low adsorbing but the microwave is high transmission, the reaction mixture melted more rapidly. Various powers were screened for the synthesizing of triazole in a domestic microwave in order to find the best power with time (monitored by TLC, AcOEt/hexane, 3:1). We found $700 \mathrm{~W}$ to be most appropriate power level for the synthesizing of triazole in a domestic microwave.

By comparision of data, it is obvious that the microwave irradiation protocol resulted in much faster reactions and significantly higher yields (Figure 1) than the thermal heating process. Furthermore, as shown in Figure 1, when using a monomode microwave, synthesized yields were higher than a 
multimode domestic microwave oven. One possible reason is that the domestic microwave oven, the pulsed irradiation (onoff duty cycles) and the resulting in homogeneity of the microwave field may lead to problems of reproducibility. Due to the non-homogenous distribution of the energy intensity throughout the cavity, some areas receive higher amounts of energy, whereas others receive less energy [13].

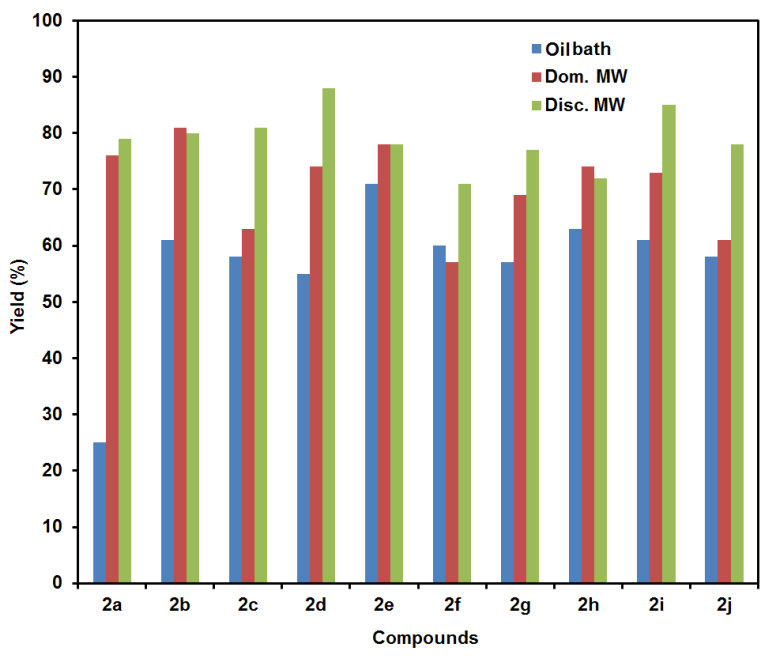

Figure 1. Compare of yields under microwave irradiation and conventional heating for compounds $\mathbf{2 a - j}$.

Here, we report our further investigations concerning the reduction of the carbonyl group in the molecule, which has been carried out to produce a corresponding hydroxyl group which possesses asymmetric carbon atom under microwave irradiation, as well as the conventional method. However, there was no reduction of carbonyl in the triazole ring but only a reduction of carbonyl, which was in the substitute group bounding N-4 nitrogen atom. Thus, in a conventional experiment, we obtained reductive products $(\mathbf{2 a - j})$ in the presence of ion exchange solid (Amberlyst- $15 \mathrm{H}^{+}$) in the aprotic solvent (THF). Amberlyst-15 $\left(\mathrm{H}^{+}\right)$is an effective assist material for reduction of hindered and unreactive ketones [14]. However, we obtained $\mathbf{3 a}-\mathbf{j}$ in the presence of alumina under microwave irradiation in the absence of solvent (Scheme 2).

Varma et al. [15] demonstrated the reduction of the carbonyl group in a solid state under microwave irradiation. Here, we synthesized 3a-j in a monomode Discover microwave synthesizer for $2 \mathrm{~min}$. at $300 \mathrm{~W}$ maximum power and in a multimode domestic microwave oven for $4 \mathrm{~min}$. at $1000 \mathrm{~W}$ power. As seen in Figure 2, generally the monomode Discover microwave irradiation protocol resulted in higher yields than the multimode domestic microwave irradiation protocol.

A comparison of the spectral data gave some information as to the nature of the products. In this context, a comparison of the IR spectral data clearly indicates the formation of compounds $3 \mathbf{a}-\mathbf{j}$ by the disappearance of the $\mathrm{C}=0$ band at about $1681 \mathrm{~cm}^{-1}$ in compounds $\mathbf{2 a - j}$, and the appearance of the $\mathrm{OH}$ band at about $3420 \mathrm{~cm}^{-1}$ and $\mathrm{C}-0$ band at about $1088 \mathrm{~cm}^{-1}$ in compounds $\mathbf{3 a}-\mathbf{j}$. In addition, in the ${ }^{1} \mathrm{H}$ NMR spectrum of $\mathbf{3 a}-\mathbf{j}$ revealed for $\mathrm{OH}$ group doublet signals at about $5.27 \mathrm{ppm}$ in the DMSO- $d_{6}$ and broad singlet signal at about $2.60 \mathrm{ppm}$ in the $\mathrm{CDCl}_{3}$, revealed for $\mathrm{CH}$ group doublet of quartets signals at about $4.76 \mathrm{ppm}$ in the DMSO- $d_{6}$ and quartet signals at about $4.90 \mathrm{ppm}$ in the $\mathrm{CDCl}_{3}$. The ${ }^{13} \mathrm{C}$ NMR spectrum of $\mathbf{3 a} \mathbf{a} \mathbf{j}$ shows a signal indicated the appearance of $\mathrm{CH}$ groups at about $69 \mathrm{ppm}$ and the disappearance of the $\mathrm{C}=0$ group at about $197 \mathrm{ppm}$ in compounds $\mathbf{2 a - j}$.

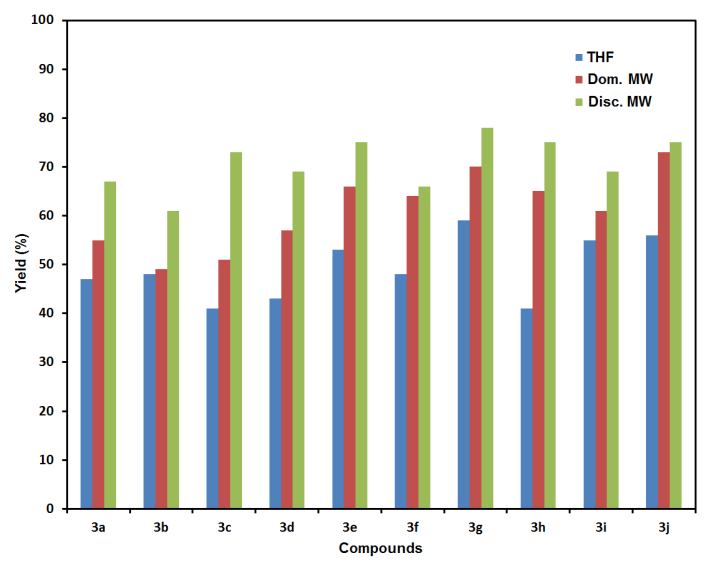

Figure 2. Compare of yields under microwave irradiation and conventional heating for compounds $\mathbf{3 a - j}$.

\section{Conclusion}

A new operationally simple, safe and environmentally benign solvent-free procedure is described for the synthesis of different $3 H-1,2,4$-triazol-3-ones and reduction of carbonyl group in these molecules under both MW and conventional heating conditions. The isolated yields of the solvent-free reactions by microwave assisted appeared to be significantly higher than the ones obtained in the corresponding reactions in oil bath. The important advantages of this procedure include ( $i$ ) operational simplicity (ease set up and work-up), (ii) safe and environmentally benign solvent free procedure (eliminating toxic organic solvents as reaction medium) (especially domestic microwave), (iii) good yield of the products with high purity (iv) mild reaction conditions, ( $\mathrm{v}$ ) good selectivity and ( $v i)$ general applicability accommodating a variety of substitution patterns.

\section{Acknowledgements}

Financial support from the Scientific and Technical Research Council of Turkey (TUBITAK) through project $108 \mathrm{~T} 221$ is gratefully acknowledged.

\section{References}

[1]. Todoulou, O. G.; Papadaki-Valiraki, A. E.; Ikeda, S. Eur. J. Med. Chem. 1994, 29, 611-620.

[2]. Rollas, S.; Kalyoncuoglu, N.; Altiner, D. S.; Yegenoglu, Y. Pharmazie 1993, 48, 308-309.

[3]. Malbec, F.; Milcent, R.; Vicart, P. J.; Bure, A. M. Heterocycl. Chem. 1984 $21,1769-1774$.

[4]. Smith, R. A.; Barbosa, J.; Blum, C. L.; Bobko, M. A.; Caringal, Y. V.; Dally, R.; Johnson, J. S.; Katz, M. F.; Kennure, N.; Kinger-Wood, J.; Lee, W. Lowinger, T. B.; Lyons, J.; Marsh, V.; Rogers, D. H.; Swartz, S.; Walling, T.; Wild, H. Bioorg. Med. Chem. Lett. 2001, 11, 2775-2778.

[5]. Bekircan, O.; Kahveci, B.; Kucuk, M. Turk. J. Chem. 2006, 30, 29-40.

[6]. Varma, R. S. Green Chem. 1999, 1, 43-55.

[7]. Lidström, P.; Tierney, J.; Wathey, B.; Westman, J. Tetrahedron 2001, 57, 9225-9283.

[8]. Kappe, C. O.Angew. Chem. Int. Ed. 2004, 43, 6250-6284.

[9]. Kahveci, B.; Ozil, M.; Mentese, E.; Bekircan, O.; Buruk, K. Russian J. Org. Chem. 2008, 44, 1816-1820.

[10]. Kahveci, B. Molecules, 2005, 10, 376-382.

[11]. Un, R.; Ikizler, A. Chim. Acta Turc. 1975, 3, 113-132.

[12]. Pesson, M.; Dupin, S.; Antoine, M. Bull. Soc. Chim. France 1962, 13641371.

[13]. Kappe, C. O.; Dallinger, D.; Murphree, S.; Practical Microwave Synthesis for Organic Chemists, Wiley, VCH, Weinheim, 2009.

[14]. Caycho, R. J.; Tellado, G. F.; Armas, P.; Tellado, M. J. J. Tetrahedron Lett. 1997, 38, 277-280.

[15]. Varma, R. S.; Saini, K. R. Tetrahedron Lett. 1997, 38, 4337-4338. 